\title{
Errors in DOI indexing by bibliometric databases
}

\author{
Fiorenzo Franceschini • Domenico Maisano • Luca Mastrogiacomo
}

Received: 15 November 2014/Published online: 23 December 2014

(C) Akadémiai Kiadó, Budapest, Hungary 2014

\begin{abstract}
DOI-i.e., Digital Object Identifier-is a character string, which univocally identifies entities that are object of intellectual property. In bibliometrics, DOIs are used for univocally identifying scientific papers. The aim of this short communication is to raise the reader's awareness of bibliometric database errors in DOI indexing, in particular, the incorrect assignment of a single DOI to multiple papers. This error is quite interesting since DOI is commonly regarded as an effective means to identify scientific articles unambiguously. For the purpose of example, a short list of DOIs, which have been wrongly assigned by the Scopus database to multiple papers, is shown. Although being relatively rare, DOI indexing errors should be considered by bibliometricians when querying bibliometric databases by DOI.
\end{abstract}

Keywords Database errors - Citation analysis · DOI indexing error · Multiple DOI assignment $\cdot$ Scopus

\section{Introduction}

Bibliometric databases-e.g., Scopus and Web of Science (WoS)—are affected by different types of errors, which may impact on citation statistics. Over the past decade, the accuracy of bibliometric databases has improved significantly, probably due to the methodical use of (semi) automatic tools for correcting errors in cited article lists by editors and database administrators (Adam 2002). However, the problem is far from being solved, as proven by the fact that bibliometric database staff constantly encourage users to report any noticed inaccuracy.

F. Franceschini $(\bowtie) \cdot$ D. Maisano $\cdot$ L. Mastrogiacomo Department of Management and Production Engineering (DIGEP), Politecnico di Torino,

Corso Duca degli Abruzzi 24, 10129 Turin, Italy

e-mail: fiorenzo.franceschini@polito.it 
Table 1 Classification of bibliometric database errors according to (Buchanan 2006)

\begin{tabular}{lll}
\hline Error type & Author errors & Database mapping errors \\
\hline Definition & $\begin{array}{l}\text { Errors made by authors when } \\
\text { creating the list of cited } \\
\text { articles for their publication }\end{array}$ & $\begin{array}{c}\text { Failure to establish an electronic link } \\
\text { between a cited article and the } \\
\text { corresponding citing articles that } \\
\text { can be attributed to a data-entry } \\
\text { error }\end{array}$ \\
Examples & $\begin{array}{l}\text { Errors in name and initials of the first } \\
\text { author } \\
\text { Errors in publication title } \\
\text { Errors in publication year }\end{array}$ & $\begin{array}{l}\text { Transtion errors } \\
\text { Cited article omitted from a cited- } \\
\text { article list }\end{array}$ \\
& Errors in volume number & Reason unknown \\
& Errors in pagination &
\end{tabular}

Several recent articles document the existence of database errors of different nature (Jacso 2006, 2012; Franceschini et al. 2013, 2014); a synthetic taxonomy of the major errors is reported in Table 1, distinguishing between author and database mapping errors (Buchanan 2006).

While author errors are generally due to lack of care of authors when creating the cited article lists, database mapping errors may originate from poor or imperfect communication between database administrators (i.e., data receivers) and editors (i.e., data providers). In fact, the administrators' requirement to quickly update bibliometric databases, whenever new data are available from editors, may sometimes conflict with indexing accuracy.

\section{Multiple DOI assignment}

In a relatively recent paper (Franceschini et al. 2014), we analyzed a set of journals indexed by Scopus and WoS, in order to estimate the rate of omitted citations, i.e., missing links between a cited paper and the corresponding citing papers. Precisely, we examined the citations indexed by Scopus and WoS, focusing on those received from journals purportedly indexed by both databases, and we showed that the citations obtained from some publishers are more likely to be omitted than those from other ones, for several reasons. The dataset included more than 27,000 articles of interest, published by 34 different journals in the field of manufacturing engineering field, with more than 112,000 citing papers issued in the time-window from 2006 to 2012.

A curious outcome of the analysis, which has not been explained in our previous paper (Franceschini et al. 2014), is the fact that bibliometric databases may sometimes mistakenly assign the same digital object identifier (DOI) to multiple papers. We recall that DOI is a character string used to univocally identify entities that are object of intellectual property (Paskin 2002). Each DOI is by definition unique and must identify one and only one entity. Since several years, DOIs are used in bibliometrics for identifying and disambiguating scientific papers. Obviously, errors in the DOI character string could distort the results of bibliometric analyses, in the case DOI is used for querying bibliometric databases. 


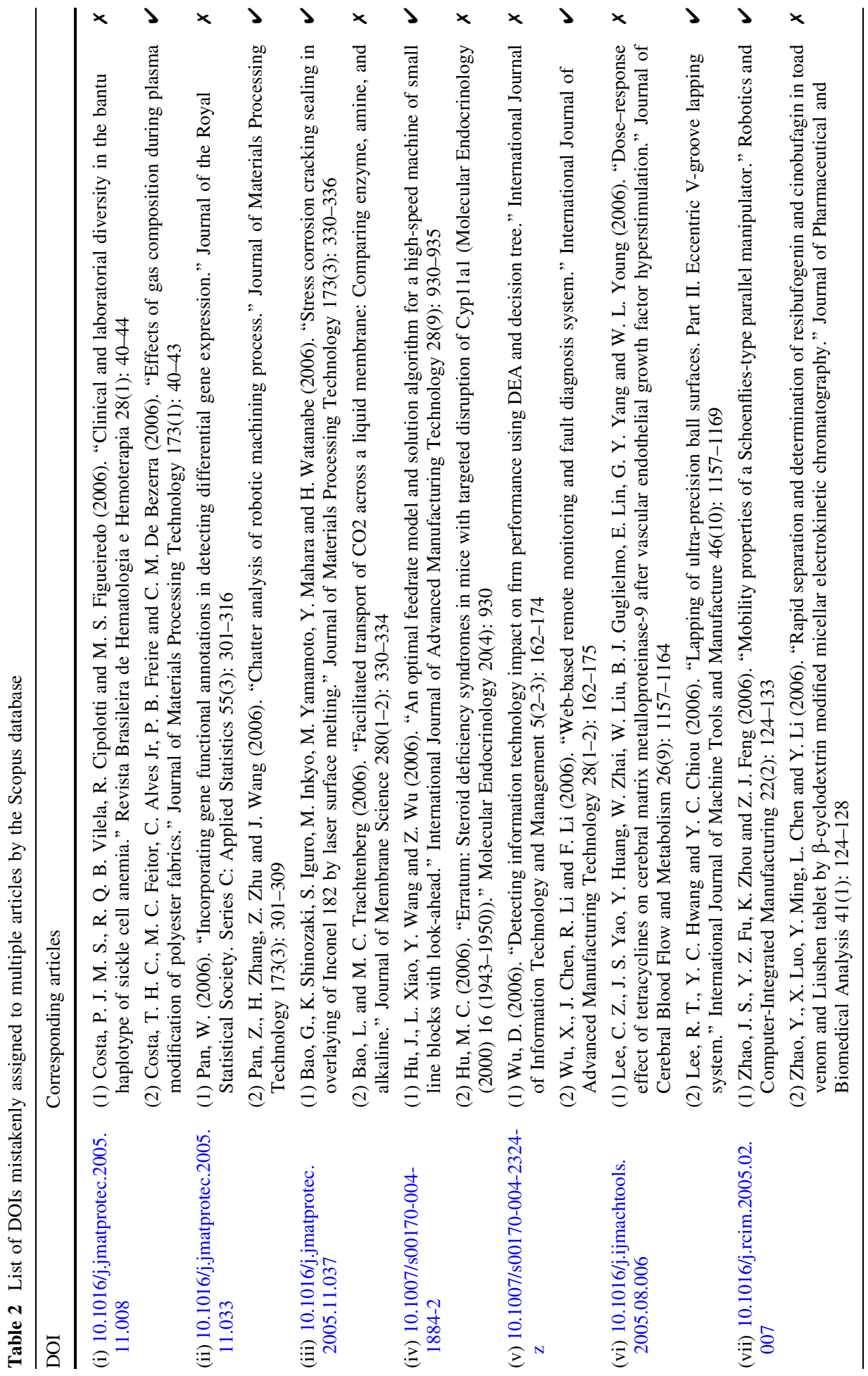




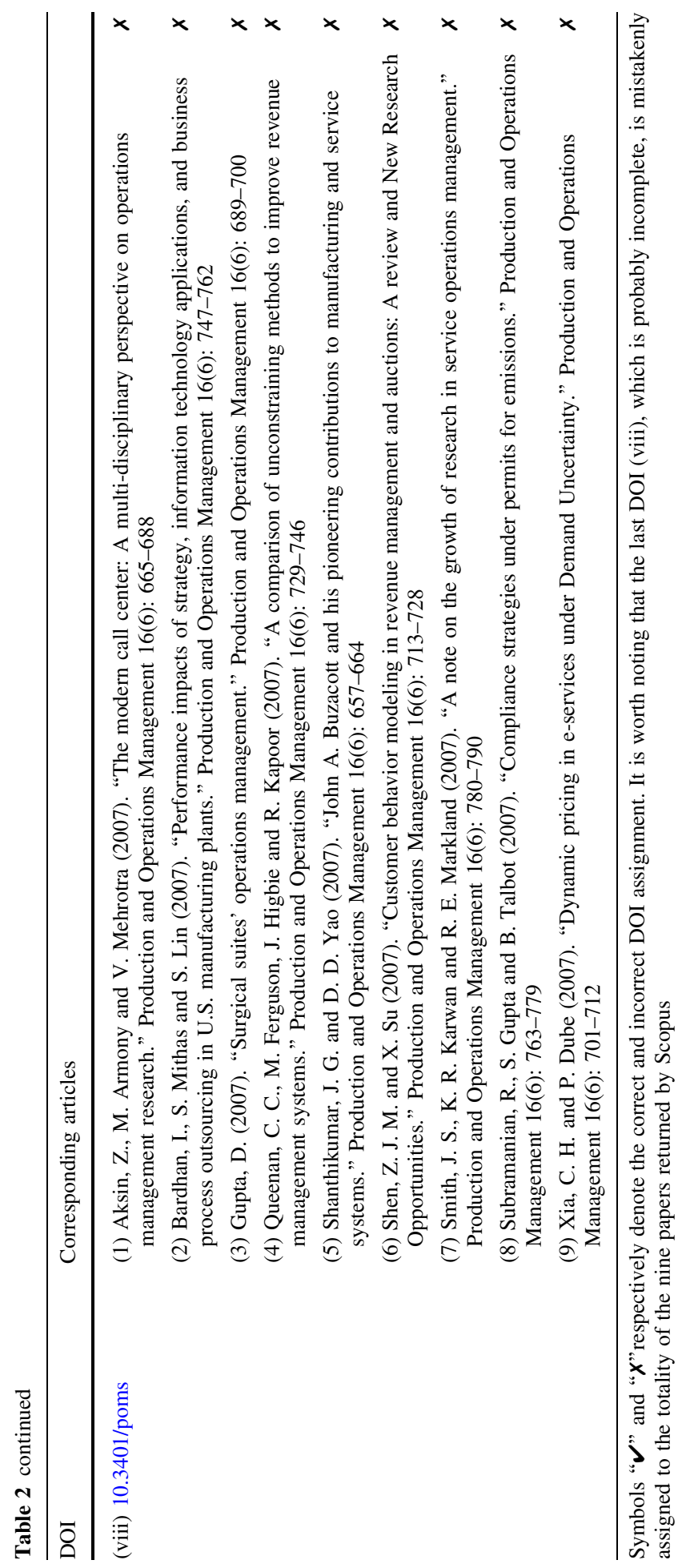




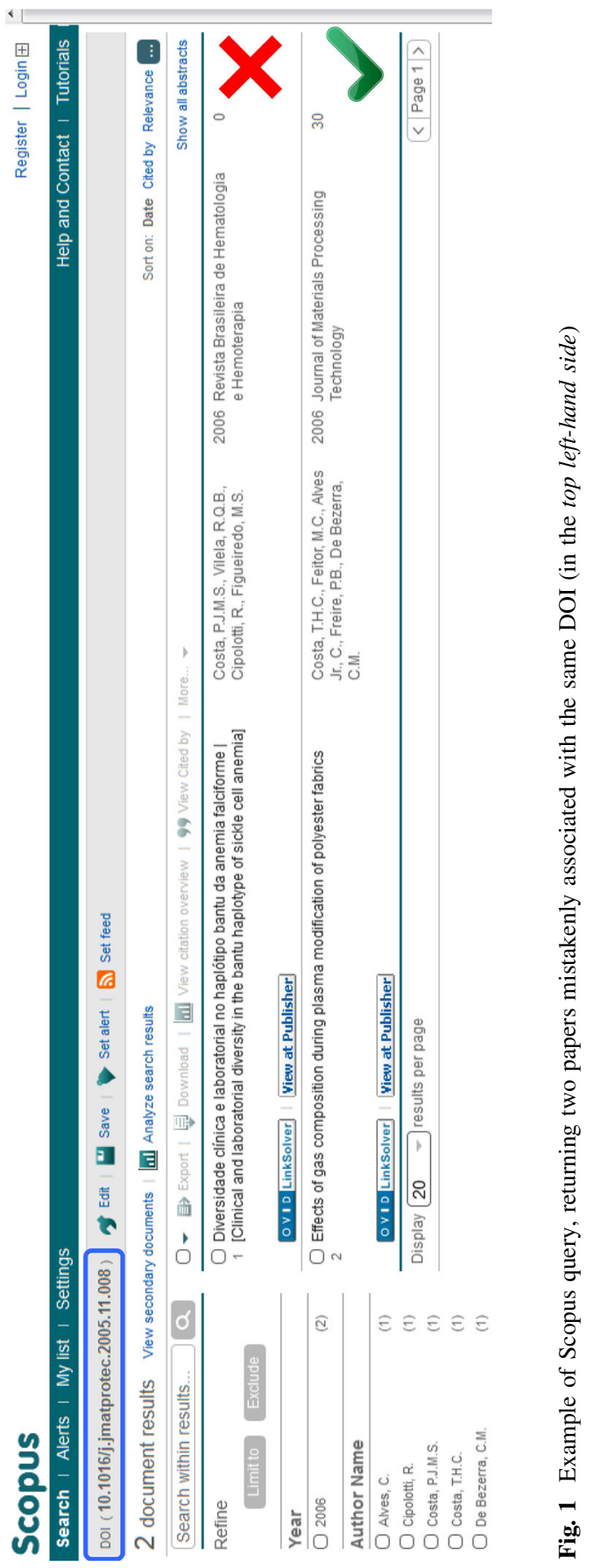


Returning to the previous discussion, Table 2 shows an example of multiple DOI assignments by the Scopus database, in which each of the eight DOIs reported (i.e., i-viii) is mistakenly assigned to two or more different articles. For the purpose of example, Fig. 1 shows the screenshot of the Scopus database response, relating to the first DOI.

It is worth remarking that the errors in Table 2 represent only a subset of those found in our analysis. Also, these errors have been detected few weeks before the submission of this short communication and they might be corrected by the database in the next future.

\section{Final remarks}

This short communication reported the existence of bibliometric database errors concerning DOI indexing. These errors are quite interesting since the DOI to a scientific paper is like the "ID card" to a person and is commonly regarded as an effective means of disambiguation. The most surprising manifestation of these errors is represented by multiple DOI assignment. Although probably being rather unusual (according to a preliminary estimation, the error rate in the Scopus database is of the order of magnitude of $1 / 1,000$ ), the fact remains that this error type could potentially influence the results of bibliometric analyses.

Future research will go into the multiple DOI assignment issue in more detail, investigating the practical reasons behind this error and providing a more accurate estimation of the error rate, for both Scopus and WoS.

\section{References}

Adam, D. (2002). The counting house. Nature, 415(6873), 726-729.

Buchanan, R. A. (2006). Accuracy of cited references: The role of citation databases. College and Research Libraries, 67(4), 292-303.

Franceschini, F., Maisano, D., \& Mastrogiacomo, L. (2013). The effect of database dirty data on h-index calculation. Scientometrics, 95(3), 1179-1188.

Franceschini, F., Maisano, D., \& Mastrogiacomo, L. (2014). Scientific journal publishers and omitted citations in bibliometric databases: Any relationship? Journal of Informetrics, 8(3), 751-765.

Jacso, P. (2006). Deflated, inflated and phantom citation counts. Online Information Review, 30(3), $297-309$.

Jacso, P. (2012). Grim tales about the impact factor and the h-index in the Web of Science and the Journal Citation Reports databases: Reflections on Vanclay's criticism. Scientometrics, 92(2), 325-354.

Paskin, N. (2002). The DOI ${ }^{\circledR}$ handbook, IDF-Intern. International DOI Foundation. 